\title{
A Literature about Electronic Game which Reproduce Contingent Graph
}

\author{
N. Rajasekar ${ }^{1}$, T. Santhosh ${ }^{2}$, B. Sasikumar ${ }^{3}$, K. Vijaya Kumar ${ }^{4}$ \\ UG Student, Department of CSE, M Kumarasaramy College of Engineering, Karur, Tamil Nadu, India ${ }^{1,2,3,4}$
}

\begin{abstract}
In this project describe a hierarchical routine for technically originating game maps using two-dimensional Markov chains. Our techniques takes collection of game maps breaks them into small chunks and platforms clustering to find a set of chunks that correspond to high-stage constructions in the practicing maps. Procedures that produce pc game at ease need game enterprise knowledge. It takes some method to mechanically study game proposal information for stage proposal from gameplay videos. In future validate the learned proposal information can be used to originate sections of game levels. In this project explored statistical procedures that could lead to generalized technical map generators. It shows rich game proposal information can be automatically analysed from gameplay videos and signified in reproductive problematic replicas. It takes Contra game for evidence. In the gameplay videos, it evaluates the method on a measure of execute ability and Quality to the real stages signified.
\end{abstract}

Keywords: Multidimensional Markov Chains (MdMC), Markov Random Fields(MRF), Hierarchical MdMCs, Procedural Content Generation.

\section{INTRODUCTION}

Creating video game maps procedurally can allow functionMapheader(Ch, Cl, PSh, PSl, Pp, Lp, PLh, PLl) playersto experience fresh and sole content. Procedural Content Generation techniquestake part game-specific proposal and domain informationinto their algorithm design and, therefore, have limitedapplicability outside of the target game. We are interested ininvestigating techniques that could be used as the foundationof domainindependent map generation approaches. In this projectdiscover machine learning methodsthat exploit dissimilar Markov Models to achieve this goal. We compare some approaches. They are MultidimensionalMarkov Chains, Hierarchical MdMCsand Markov Random Fields. The collection of training maps for the target domain is the only requirement to access.By Learning the above techniques, this project intendto abstract latent schemeinformation fromskilled created mapsthat else it needs to be provided by designer.

\section{PROPOSED ARCHITECTURE}

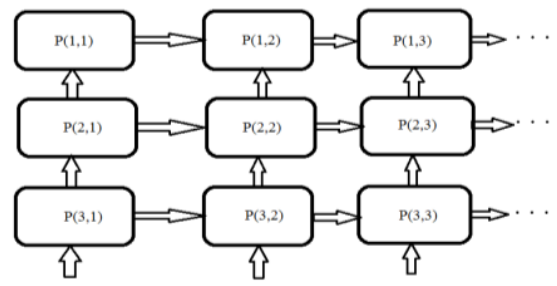

Fig.1. Linking of maps in Multi-dimensional

\section{PROPOSED ALGORITHM}

C - Gap, PS -Primary State, Pp=Player position, Lp Left portion, PL-PLatform

$\mathrm{Mh}=\mathrm{Map}(\mathrm{Ch}, \mathrm{PSh}, \mathrm{Pp}, \mathrm{PLh})$

$\mathrm{Ml}=$ Empty Map

forPpl $€$ coordinates $(\mathrm{Ml})$

Pph $€$ coordinates( $\mathrm{Mh})$ do

for each ps $€$ PSl do ifTileHeader(Mh, Ml, Ppl, Pph,

$\mathrm{Cl}, \mathrm{ps}, \mathrm{Lp}, \mathrm{PLl})$ then

break

end if

end for

end for

return $n$

end function

functionTileHeader(Mh, Ml, Ppl, Pph, Cl, ps, Lp, PL)

if $\mathrm{C}<0 \mathrm{~V}$ outSideMap(cood, Ml) then

Return true

end if

PL* $=$ PL

Con $=\operatorname{config}(\mathrm{Ml}, \mathrm{Ppl}, \mathrm{ps})$

$\mathrm{Th}=\mathrm{Mh}[\mathrm{Pph}]$

if Con is hiddenformal then

Return false

else

ttestedallowing to Lp[ps][th][PL*|Con] $\mathrm{Ml}[\mathrm{Ppl}]=\mathrm{t}$

end if

while $\sim$ TileHeader(Mh, Ml, Ppl + 1,Pph , C-1, ps, Lp, PL*)

do

$\mathrm{PL}^{*}=\mathrm{PL} \backslash \mathrm{t}$
If PL* $!=0$ then

Return false

Else 


\author{
Lp[ps][Th](PL*|Con)

$$
\mathrm{ml}[\mathrm{Ppl}]=\mathrm{t}
$$ \\ end while \\ return true \\ end function

\section{end if}

t sampled according

to

\section{ANALYTIC RESULT}

\section{PLAYABILITY}

Below table shows the good execute ability results attained by every model in each domain. It is taken for statistical significance testing using playability results our replicas and for Contra.

HMdMC and clustering based HMdMC significantly overtake the MdMC and MRF replicas. It implements that signifying maps at many resolutions allow for more precisereplicas of the charts. It originate that the MRF was talented to give significantly mostexecutable maps for Contra than any of the other replicas. For Tank the MdMC and HMdMCreplicas were talented to givepointedly more executable maps.

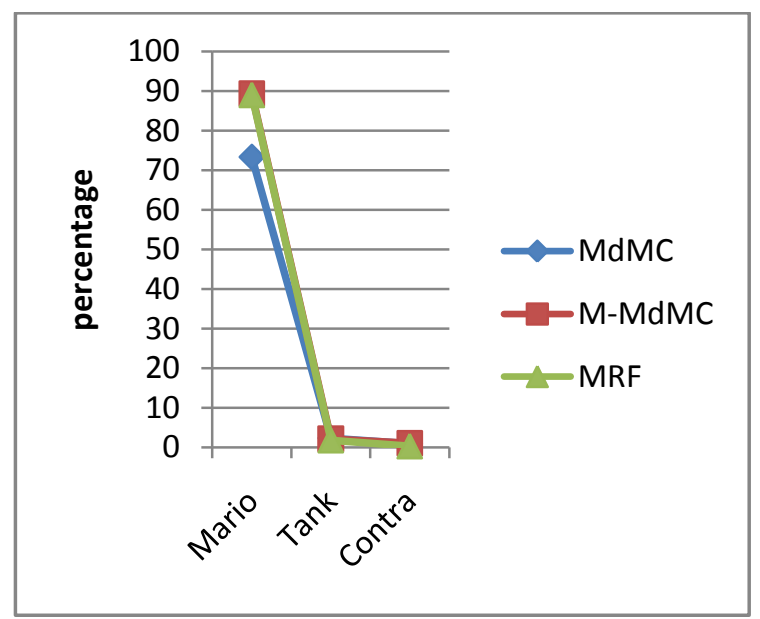

\begin{tabular}{|l|l|l|l|}
\hline APPROACH & Mario & Tank & Contra \\
\hline MdMC & $73.3 \%$ & $2.3 \%$ & $0.9 \%$ \\
\hline M-MdMC & $89.3 \%$ & 2.1 & $1.0 \%$ \\
\hline MRF & $89 \%$ & $1.8 \%$ & $0.3 \%$ \\
\hline
\end{tabular}

TIME: (milli seconds per stage)

Below table shows the mediocre no of time (in milliseconds). It takes to example a fresh stage in every domain using every of the replicas. It attained these values by practicing the model and sampling 100 levels using the baseline configuration. It noted the no of time to example those 100 stages. Middling the calculated for one stage. The MRF predicted to be the lowest as for examples by reordering tiles in a stage for hundreds of thousands of loops. Consequently, it checks over sole map more times before concluding, whereas the HMdMC and MdMCreplicas only predicted go over the stage a sometimes.

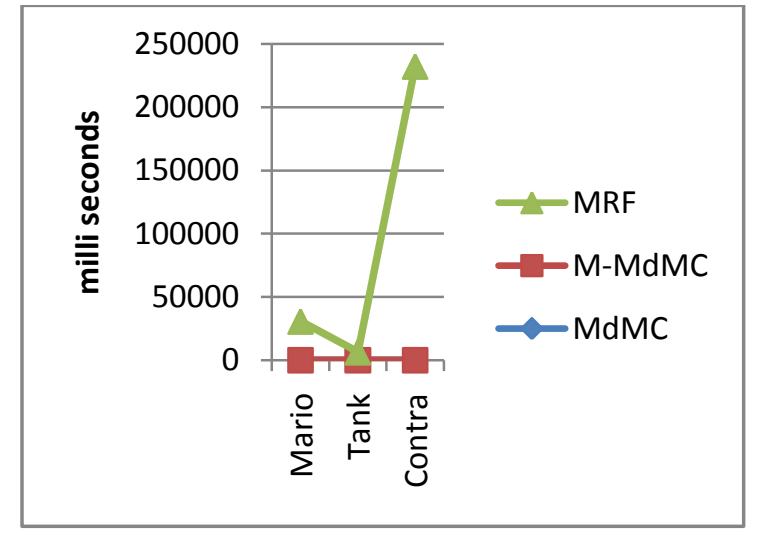

\begin{tabular}{|l|l|l|l|}
\hline APPROACH & Mario & Tank & Contra \\
\hline MdMC & 21.9 & 7.02 & 12.8 \\
\hline M-MdMC & 15.17 & - & - \\
\hline MRF & 30728 & 6451 & 232137 \\
\hline
\end{tabular}

\section{EXISTING SYSTEM}

The originate computer game contents need game proposal information by algorithms. It presents a method to automatically attain game proposal informationfor stage proposal from game play videos. But it is unable to observe the human originality that gives the most stimulating stage proposals. These are regular for their particular domain more specific. Our methodincludes analysing video of people executing a game to find the design of shapes of sprites and utilizing mechanism attaining to build a problematicreplicate of sprite assignment. It displays ironic game suggestion information can be mechanically studied from gameplay videos and signified in reproductive problematic replicas. Markov chain has been used for monitor the plat former stages as continues process. Gameplay videos, it evaluates method on a measure of execute ability and designate comparing to the real stages as indicated.

\section{IMPLEMENTATION}

In our project we excluded the algorithm which creates game levels on the run time. So based on the difficulty stage game particles are placed. This method tested with our replicas by practicing them on expert compressed maps from Mario, tank, \& Contra. Then sampling of maps for every of domains. This delivers useful visions into the assetsplusfaintness of Markov chain method.To stage generation in order to monitor further work in this region, and highlights the request for assessingprotocols using more than singleaimarea.

\section{RELATED WORKS}

Technical stage generation is the problem of generating high-quality game levels automatically. Existing stage generation schemes typically hire rule centred techniques and express optimization difficulties, where an expert 
proposal encrypts domain precise information as a set of instructions, restraints, and neutral functions. (Matthew Guzdial, Mark Riedl-2016)Manually creating maps for games is expensive and time consuming. Delegating map generation to an algorithmic process can save developers time and money, or even allow novel forms of gameplay. (Sam Snodgrass, Santiago Ontanon) [2]

Generating video game gratified algorithmically (PCG), allows player to practise fresh and exclusive data. Furthermost PCG research focuses on domain specific approaches. (Sam Snodgrass)[6]

Video games examine the use of Long Short-Term Memory recurrent neural networks (LSTMs) for the purpose of generating levels trained from a corpus of Super Mario Bros. It analyse a number of different data representations and how the generated levels fit into the space of human authored Super Mario Bros.(Adam J. Summerville, Michael Mateas)[7]

Aesthetically pleasing manner, a moral technical stage originator must gratify problem restraints. Current methods to technical stage origination schemes for platformers often focus on the restraints implemented, and limit varies output. (Peter Mawhorter, Michael Mateas)[11]

\section{CONCLUSION}

This project explored the automatic decisions on different places and different time. It has tested between many video games for playability. Any levels it proceeds with artificial method to do actions. Primary test was taken in the video game Mario. Here every particle in the game was generated by using the replacement algorithm. Sometimes this can be cut down meaningfully on the required for practicing data from the aimareaand also grant for the selection of stages that may not have been likely without the out of areapracticing data. Our model requires no manual annotation and can extract all informationin an unsupervised fashion from gameplay video. Through a user study, it find strong evidence that our model captures style and underlying proposal of an exemplar set better than the current state of the art. Arithmeticaltechnical content generation approaches have been gaining attention recently, but little has been done towards generalized content generators.Atechniquefor information remains a hugemilestone in technical content creation then in general video game proposal. It contains a newmethod to predefinedattaining of computer game proposalinformationby gameplay video, with a precise focus on stageproposal knowledge.

\section{REFERENCES}

[1] M. Guzdial and M. Riedl, "Toward game stage generation from gameplay videos,"

[2] S. Snodgrass and S. Onta n'on, "Experiments in map generation using markov chains,"

[3] P. Mawhorter and M. Mateas, "Technicalstage generation using occupancy-regulated extension," in Computational Intelligence and Games (CIG)
[4] L. R. Rabiner, "A tutorial on hidden markovreplicas and selected applications in speech recognition,"

[5] Matthew Guzdial, Mark Riedl," Game Level Generation from Gameplay Videos",

[6] Sam Snodgrass," General Statistical Approaches to Technical Map Generation"

[7] A. Summerville and M. Mateas, "Super mario as a string: Platformerstage generation via lstms,"

[8] S Saravanan, V Venkatachalam " Enhanced bosa for implementing map reduce task scheduling algorithm" International Journal of Applied Engineering Research,Vol 10(85),pp60-65,2015.

[9] S.Swathi "Preemptive Virtual Machine Scheduling Using CLOUDSIM Tool", International Journal of Advances in Engineering, 2015, 1(3), 323 -327 ISSN: 2394-9260, pp:323-327.

[10]. S Saravanan, V Venkatachalam, S Then Malligai "Optimization of SLA violation in cloud computing using artificial bee colony"2015, 1(3), 323 -327 ISSN: 2394-9260, pp:410-414.

[11] P. Mawhorter and M. Mateas, "Procedural level generation usingoccupancy-regulated extension," in Computational Intelligence andGames (CIG), 2010 IEEE Symposium on. IEEE, 2010, pp. 351-358. 